\title{
AVALIAÇÃO PÓS OCUPAÇÃO - ESTUDO DE CASO NO CAPS II DA CIDADE DE BALNEÁRIO CAMBORIÚ, SC
}

\author{
CAMPOS, Rafael Alves de \\ Universidade do Vale do Itajaí, e-mail: arqrafaelcampos@gmail.com \\ JEFfE, Ana Paula Magalhães \\ Universidade do Vale do Itajaí, e-mail: arq3ana@gmail.com \\ LOCH, Márcia do Valle Pereira \\ Universidade do Vale do Itajaí, e-mail: marcia.loch@hotmail.com \\ MOLIN, Rodrigo Dal \\ Universidade do Vale do Itajaí, e-mail: rodrigodalmolin76@gmail.com \\ PELLIZZARO, Julia Perin \\ Universidade do Vale do Itajaí, e-mail: juliaperin40@gmail.com \\ RIBEIRO, Raryana Fernanda \\ Universidade do Vale do Itajaí, e-mail: raryanaribeiro $1 @ g m a i l . c o m$ \\ SESSEGOLO, Maria Eduarda Donatto \\ Universidade do Vale do Itajaí, e-mail: dudadsesse@gmail.com
}

\begin{abstract}
RESUMO
Este artigo apresenta parte dos resultados obtidos através da pesquisa realizada pelo Grupo de Extensão Arquitetura e Cidades Saudáveis, para a requalificação da edificação do Centro de Atenção Psicossocial (CAPS II), localizado na cidade de Balneário Camboriú - SC. A metodologia aplicada consiste na Avaliação Pós Ocupação, por meio de uma abordagem quali/quantitativa. Alguns dos instrumentos de APO utilizados para coleta dos dados foram: Observações Assistemáticas; Entrevistas semiestruturadas; e Walkthrough. Para o tratamento dos resultados foram elaboradas uma matriz de descobertas e uma de recomendações, que serviram como base para projeto de requalificação dos ambientes da edificação que abriga o CAPS II. Os resultados apontaram que a unidade possui diversos conflitos de fluxo, setorização, subdimensionamento de ambientes, falta de acessibilidade e layout inadequado para 0 desenvolvimento das atividades.
\end{abstract}

Palavras-chave: Avaliação Pós Ocupação, Centro de Atenção Psicossocial, Requalificação.

\begin{abstract}
This paper presents part of the results obtained through the research developed from the Architecture and Healthy Cities Extension Group for the requalification of the Psychosocial Attention Center (CAPS II) building, located in the city of Balneário Camboriu - SC. The applied methodology consists of the Post-Occupancy Evaluation, through a qualitative and quantitative approach. Some of the APO instruments used to collect the data were unsystematic observation, semi-structured interviews and walkthrough. For the treatment of the results were elaborated a Matrix of Discovery and a Matrix of Recommendations. These instruments gave basis for the requalification project of the CPAS II building. The results indicated that the internal spaces has several conflicts of flow, sectorization, undersizing, lack of accessibility and inadequate layout for the development of activities.
\end{abstract}

Keyowrds: Post-Occupancy Evaluation, Psychosocial Attention Center, Requalification.

CAMPOS, R. A.; JEFFE, A. P. M.; LOCH, M. V. P.; MOLIN, R. D.; PELLIZZARO, J. P.; RIBEIRO, R. F.; SESSEGOLO, M. E. D. Avaliação pós-ocupação - estudo de caso no CAPS II da cidade de Balneário Camboriú, SC. In: SIMPÓSIO BRASILEIRO DE QUALIDADE DO PROJETO NO AMBIENTE CONSTRUÍDO, 6., 2019, Uberlândia. Anais... Uberlândia: PPGAU/FAUeD/UFU, 2019. p. 1124-1131. DOl https://doi.org/10.14393/sbqp19102. 


\section{INTRODUÇÃO}

Segundo portal do ministério da Saúde, a depressão (CID 10 - F33) provoca ausência de prazer em coisas que antes faziam bem e grande oscilação de humor e pensamentos, que podem culminar em comportamentos e atos suicidas. O tratamento é feito com auxílio médico profissional, por meio de medicamentos, e acompanhamento terapêutico conforme cada caso. $O$ apoio da família é fundamental. A depressão é uma doença que afeta a saúde mental de milhares de brasileiros, e o aumento expoente de casos de depressão, síndromes de pânico, melancolias e demências em diversos níveis floresce de um cenário em que o tema saúde mental segue como tabu. $O$ reconhecimento da concretude das doenças mentais e seu impacto avassalador na sociedade contemporânea é tão emergente quanto trazer a luz da ciência ao tema lugares adequados para promover a saúde da mente.

Com a Reforma Psiquiátrica no Brasil, movimento que acabou com o modelo manicomial, atualmente a assistência à saúde mental acontece através de uma rede de assistência psicossocial. Inseridos nesta rede estão os Centros de Atenção Psicossocial - CAPS, equipamento que propõe nova abordagem na saúde mental, com a humanização do tratamento e ressocialização dos usuários (JEFFE, 2005).

Segundo a portaria $n^{\circ} 336$ do Ministério da Saúde as unidades devem ser independentes de outras instalações de saúde ou educação, com acesso privativo e equipe própria. Nos dias de hoje, existe a necessidade e a demanda de inserir no âmbito da Reforma Psiquiátrica, o planejamento da infraestrutura física e urbana destes estabelecimentos. É recorrente que os CAPS estejam instalados em estruturas que não foram edificadas para este tipo de uso, o que colabora para a criação de espaços que não atendem as demandas técnicas e as expectativas de seus usuários.

De acordo com o ofício $n^{\circ} 076$ da Secretaria Municipal de Saúde e Saneamento de Balneário Camboriú, o CAPS II está localizado em uma edificação que dificulta o atendimento de saúde aos seus usuários. A partir disto surgiu a demanda de desenvolver projeto e ações de melhoria das condições espaciais deste estabelecimento de saúde.

Para atender a esse objetivo, este estudo apresenta a avaliação da edificação do CAPS II, realizada por meio de instrumentos de Avaliação Pós Ocupação (APO), através de uma abordagem quali/quantitativa. O presente artigo busca apresentar parte dos resultados obtidos por meio de uma pesquisa realizada no ano de 2018, pelo Grupo de Extensão Arquitetura e Cidades Saudáveis, da Universidade do Vale do Itajaí.

O lugar de cura requerido para os espaços utilizados pelos CAPS requer estudos aprofundados, vinculando as ciências arquitetura e saúde para a constituição de balizadores projetuais que componham com as práticas de saúde e assim resulte em efetiva ação de cura de saúde mental. O ambiente deve transmitir a sensação de proteção, acolhimento e resolução aos sujeitos que nele estão inseridos, devendo ser consideradas também a privacidade e a individualidade, além de trabalhar os espaços com elementos que interagem com o ser humano diariamente, como, por exemplo, a iluminação, o som, o cheiro e a cor (BRASIL, 2004). 


\section{METODOLOGIA APLICADA}

A metodologia utilizada para o desenvolvimento desta pesquisa foi a Avaliação Pós Ocupação (APO) (RHEINGANTZ et al., 2008), com abordagem qualitativa. A pesquisa foi aplicada com o corpo técnico e com usuários do CAPS II, que permitiu a percepção das problemáticas quanto a fluxos, layout, tratamento acústico, conforto térmico, acessibilidade e paisagismo.

Foram realizadas observações assistemáticas que apontaram conflitos de fluxos, entrevistas semiestruturadas e fotografias. Essas etapas geraram um roteiro e uma tabela para coleta de dados como demonstra a Figura 1. A partir de então, realizou-se a avaliação de desempenho da edificação que levou em consideração as condições acerca da estrutura, do conforto térmico e acústico, layout, distribuição das salas e percepções dos colaboradores.

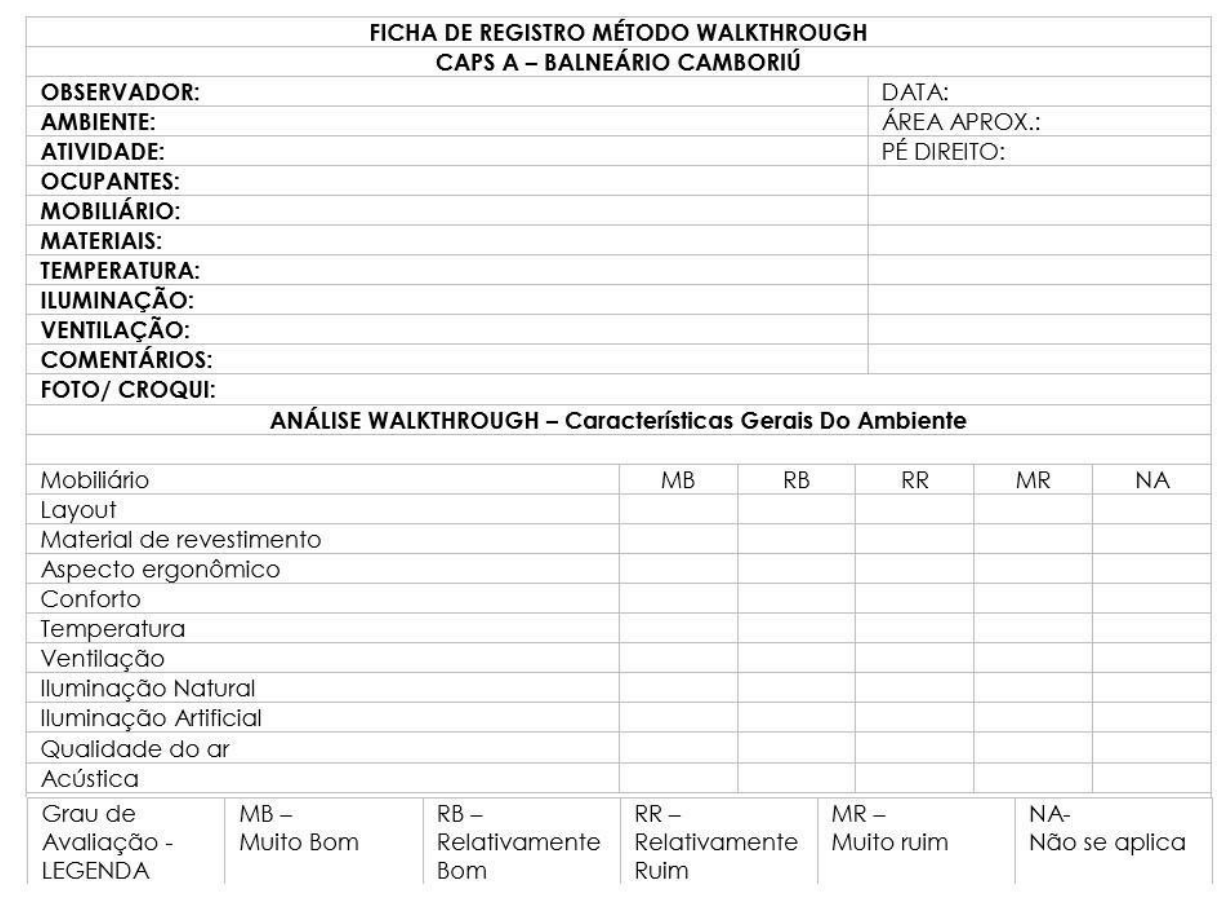

Figura 1 - Ficha do desempenho do ambiente Fonte: Autores (2018)

Outro instrumento utilizado para coleta dos dados foi o walkthrough, que consiste em uma análise que combina simultaneamente uma entrevista com uma observação, possibilitando a identificação de aspectos negativos e positivos dos ambientes analisados. É um método muito eficaz, pois revela a percepção dos entrevistados sobre o espaço (RHEINGANTZ, et al., 2008). Foram realizadas duas aplicações do método com a participação de duas colaboradoras: uma enfermeira e uma psicóloga. Foi realizado um percurso pelo CAPS e durante o trajeto, a entrevistada foi conduzida a observar questões relevantes a pesquisa, por meio da aplicação de um formulário de análise do ambiente desenvolvido pela equipe de pesquisadores, além de realizar uma entrevista semiestruturada. 
Na imagem seguinte (Figura 2), pode-se observar o percurso realizado, onde os círculos demonstram os ambientes com maior concentração de pessoas em atividades distintas.

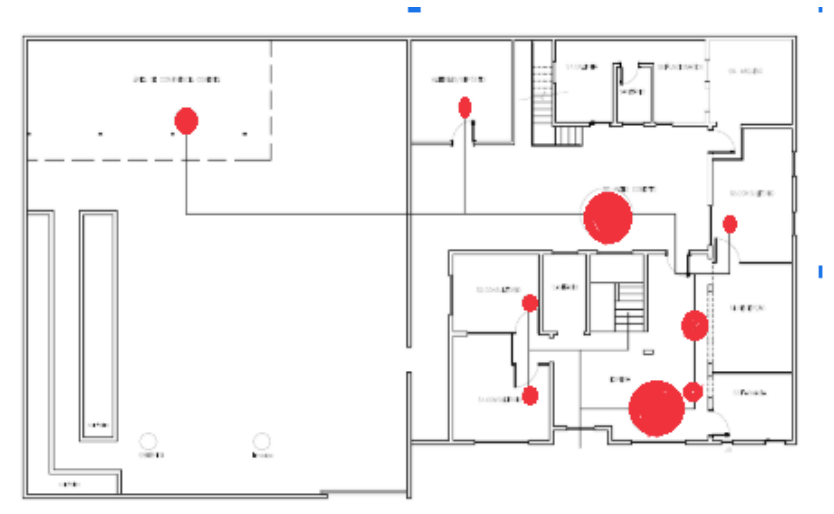

Figura 2 - Percurso realizado no Walkthrough Fonte: Autores (2018)

Os dados coletados nas etapas descritas anteriormente foram compilados e organizados em duas matrizes, uma de descobertas e outra de recomendações. A primeira é o tratamento dos dados em um único documento, com o objetivo de apresentar graficamente de forma clara e resumida as principais descobertas a partir da planta de setorização. Concebida por Helena Rodrigues e Isabelle Soares, "é um instrumento de análise que permite identificar e comunicar graficamente as descobertas, especialmente aquelas relacionadas com: (a) as adaptações e improvisações decorrentes de falhas de projeto ou de execução; (b) a incompreensão e o desconhecimento dos seus diversos grupos de usuários, que dificultam a operacionalidade necessária no dia-a-dia de um ambiente" (RHEINGAINTZ et al., 2008, p.12).

A Matriz de Recomendações é a segunda etapa da matriz de descobertas, sendo o resumo da mesma e com informações que indicam possíveis ações, um partido a ser seguido para formulação de propostas físicas de intervenção no espaço-objeto de estudo, para curto, médio ou longo prazo.

\section{ANÁLISE DOS RESULTADOS}

A análise do CAPS II, apontado após a aplicação dos instrumentos de avaliação, indicam os seguintes conflitos: fluxos, falta de acessibilidade, falta de isolamento acústico, instalações elétricas inadequadas, infiltrações, falta de ventilação e luz natural e falta de privacidade.

De acordo com os resultados obtidos verificou-se que a sala de espera está localizada em local inadequado e a disposição do mobiliário dificulta a realização das atividades. Ainda neste ambiente acontecem os atendimentos da recepção e farmácia, além do fluxo de usuários, acarretando em conflitos de atividades e fluxo. O ambiente é subdimensionado e têm mobiliário desconfortável para as longas esperas dos atendimentos. Afora estas questões técnicas, também pode-se perceber que o espaço como um todo transmite o sofrimento que os usuários levam consigo devido à falta de humanização do lugar, que pode ser observado na Figura 3. 


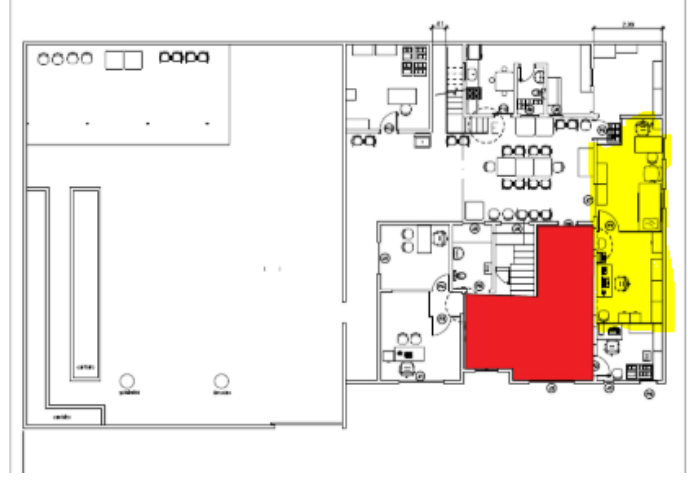

- em vermelho, a sala de espera

- em amarelo, recepção e enfermagem

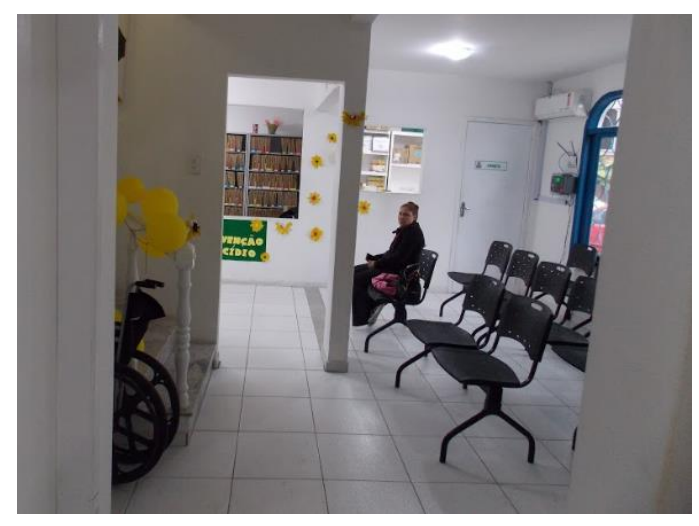

- layout e espacialidade da sala de espera

Figura 3 - Localização em planta da sala de espera e vista da sala de espera Fonte: Autores (2018)

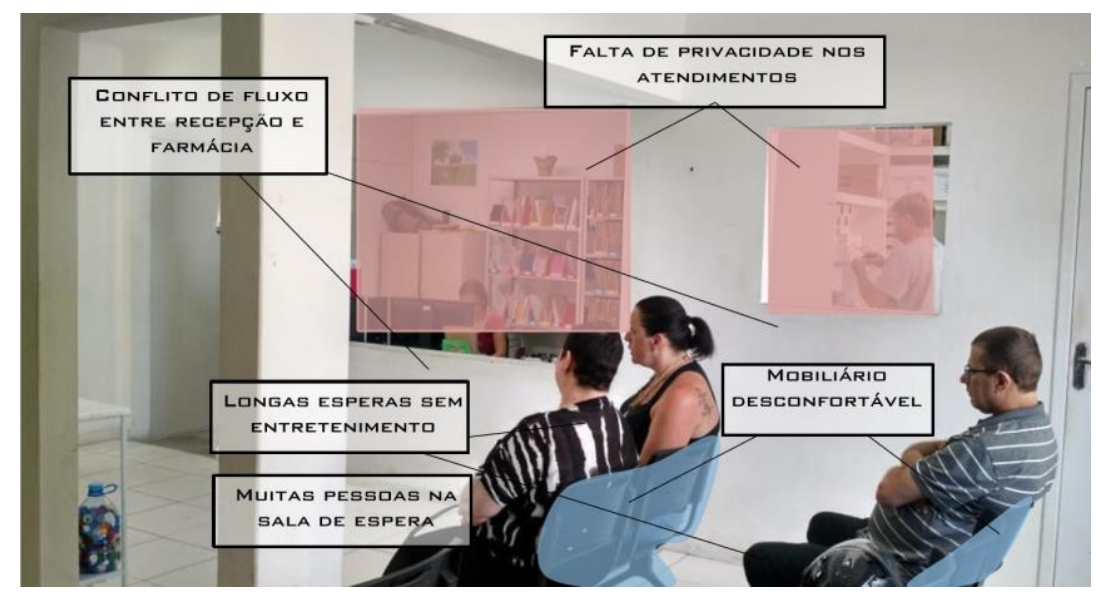

a) Sala de espera

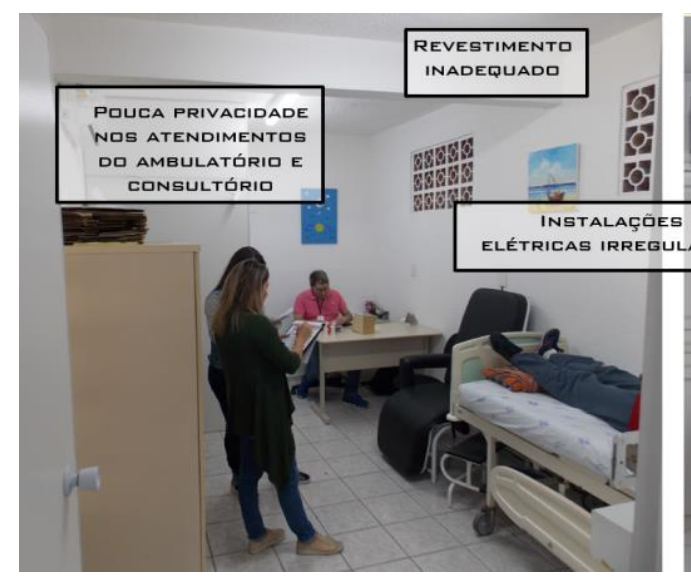

b) Recepção

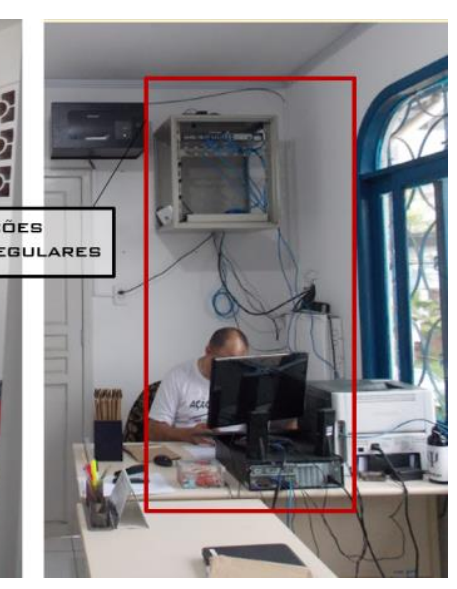

c) Farmácia

Figura 4 - Análises realizadas após aplicação das ferramentas de APO Fonte: Autores (2018)

Outras questões pertinentes sobre a estrutura são: a parede da recepção possui uma grande abertura e dificulta 0 atendimento de pessoas com mobilidade reduzida, cadeirantes e impossibilita a instalação de um móvel com atendimento individualizado. Pode-se observar como a estrutura e as adequações realizadas na unidade impedem a acessibilidade, conforme 
apontado na Figura 4. Outra situação que se destacou foi o fato de a enfermagem e consultórios serem separados por divisórias simples, não possuírem isolamento acústica, não existindo ventilação natural e com instalações elétricas inadequadas. Estes ambientes necessitam de revestimento acústico por serem espaços que tratam de intimidades dos usuários, tendo, por muitas vezes, o som propagado pelo espaço.

Os principais resultados obtidos foram compilados em uma matriz de descobertas. Como pode-se observar na Figura 5, a recepção e a farmácia não oferecem nenhuma privacidade devido à localização na circulação geral, tendo falta de mobiliário adequado ao atendimento.

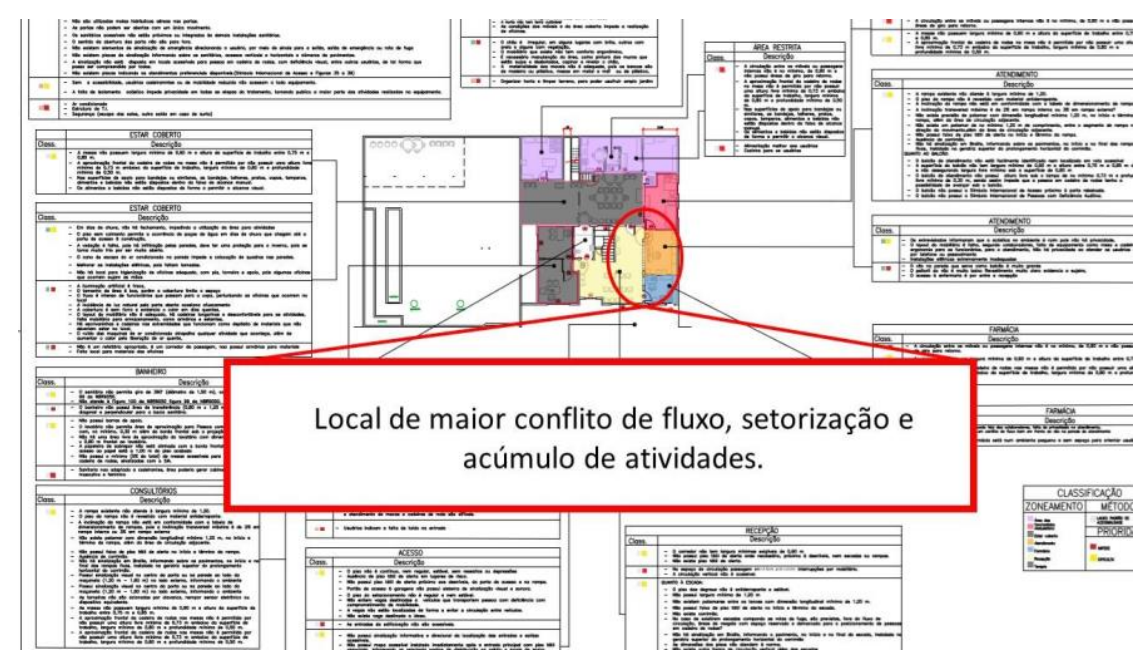

Figura 5 - Matriz de Descobertas -

Fonte: Autores (2018)

Após as aplicações e processamento dos resultados da pesquisa, o grupo de extensão iniciou algumas intervenções com objetivo de promover melhorias no espaço a curto prazo e a apropriação dos usuários com a edificação. Uma delas foi a pintura interna dos ambientes onde existe maior interação com os usuários, com o intuito que envolver os colaboradores na mudança do local, promovendo melhoria na qualidade ambiental e organizar a dinâmica espacial, humanizando o espaço existente.

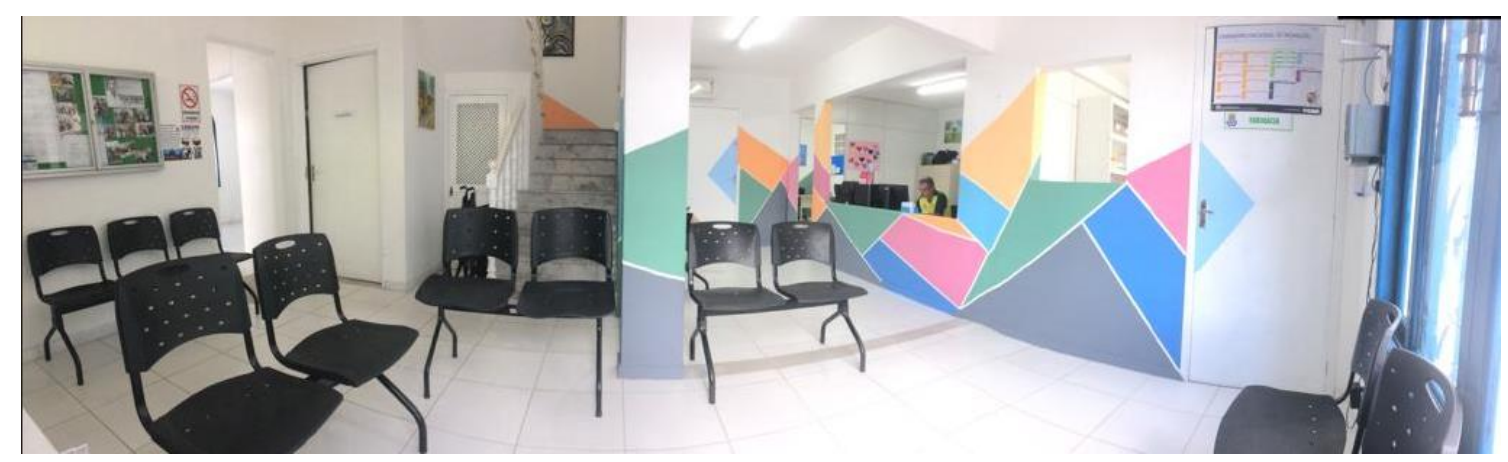

Figura 6 - Recepção e sala de espera após intervenção -

Fonte: Autores (2018)

Segundo Farina, Perez e Bastos (2006), as cores influenciam o ser humano no caráter psicológico e fisiológico, "criando alegria ou tristeza, exaltação ou depressão, atividade ou passividade, calor ou frio, equilíbrio ou desequilíbrio, ordem ou desordem etc." Elas podem imprimir sensações e reflexos, pois cada 
uma tem uma vibração determinada em nossos sentidos e atuam como estimulante ou perturbador na emoção, na consciência e em nossos impulsos e desejos.

Para atividade anteriormente citada, uma paleta de cores foi desenvolvida pelos pesquisadores e a composição das cores foi escolhida pelos colaboradores. Abaixo segue uma imagem após a intervenção das cores e alteração do layout na sala de espera (Figura 6).

\section{CONSIDERAÇÕES FINAIS E RECOMENDAÇÕES}

O primeiro acesso de pessoas da comunidade que chegam ao CAPS ocorre por meio de um atendimento denominado acolhimento, a portaria $n^{\circ} 336$ do Ministério da Saúde indica que os CAPS devem representar um segundo lar aos usuários, que não devem estar inseridas em hospitais ou universidades, e sim, preferencialmente em casas. Deseja-se que os espaços dos CAPS representem um ambiente receptivo, que deem conta de superar o tecnicismo indiferente dos ambientes de saúde, por um substitutivo caloroso e acolhedor.

Contudo, o comumente ocorrido é a indicação que as unidades optem por instalar-se em casas pré-existentes. Entretanto, constata-se que as casas acabam por não priorizar o conjunto de normativas técnicas necessárias para atender as unidades de saúde, como acessibilidade universal, materialidade adequada, fluxos que atendam as múltiplas funções de um lugar de saúde, entre outros pontos.

O espaço físico do CAPS II de Balneário Camboriú apresenta muitos conflitos pelo fato da edificação ser alugada e adaptada, o que acaba não conseguindo suprir as demandas técnicas da unidade. A sua ocupação e as adaptações ocorreram sem um planejamento adequado, o que prejudica e restringe a realização de atividades.

Detecta-se que faltam normas e legislações mais específicas para a elaboração de projetos para os espaços físicos de novos centros de atenção psicossocial. Percebe-se a necessidade da criação de um roteiro mínimo, com parâmetros adequados para a instalação deste tipo de estabelecimento de saúde. O uso de casas alugadas sem as devidas adaptações, acabam por criar espaços sem qualidade ambiental, que atendam de forma adequada as atividades e seus usuários.

Como ação a curto prazo, foram propostas e realizadas oficinas de requalificação com participação dos colaboradores e usuários que além de trazer vitalidade e humanização para o CAPS, também ajudam na apropriação e zelo do espaço pela comunidade que frequenta a unidade. As oficinas ampliam a percepção do usuário quanto a elementos que compõem - lugar que ele ocupa e tornam-se mais um recurso da terapia ocupacional, colaborando no seu processo de cura.

Percebe-se a carência de pesquisas e referenciais sobre lugares projetados para promover a saúde em seu aspecto amplo, ainda mais referenciais teóricos para subsidiar projetos de lugares para curar a mente. Acrescenta-se aqui que estas pesquisas precisam ser realizadas de forma sistêmica, considerando tanto aspectos arquitetônicos como de procedimentos da equipe de saúde mental, relacionando e integrando pelo menos estas duas 
grandes áreas a fim de verificar as necessidades básicas e complementares desta unidade de saúde.

Por fim, conclui-se que o ideal para estes equipamentos sejam espaços livres e aconchegantes, com a intenção de quebrar a frieza dos ambientes hospitalares e torná-los acolhedores e voltados a promover a cura e saúde dos usuários. O espaço do CAPS deve representar um lugar de abrigo para os seus usuários, e por isso é preciso fomentar a apropriação espacial pelos mesmos, buscando identificar as suas necessidades e as melhores alternativas para atendê-las, propondo atividades de forma colaborativa para que todos os envolvidos, usuários e técnicos possam intervir na qualidade do espaço como ferramenta terapêutica.

\section{REFERÊNCIAS}

BRASIL. Ministério da Saúde. Humanizasus: ambiência. Brasília: Editora do Ministério da Saúde, 2004.

EBC - Empresa Brasil de Comunicação. Saúde mental: transtornos atingem cerca de 23 milhões de brasileiros. 2013. Disponível em:

<http://www.ebc.com.br/noticias/saude/2013/05/saude-mental-em-numeroscerca-de-23-milhoes-de-brasileiros-passam-por>. Acesso em: 19 fev. 2019.

FARINA, M.; PEREZ, C.; BASTOS, D. Psicodinâmica das cores em comunicação. 5. ed. São Paulo: Blücher, 2006.

JEFFE, A. P. M. Comunidade Terapêutica para Tratamento da Dependência

Química: Investigação da Arquitetura como um dos Condicionantes Terapêuticos. TCC em Arquitetura e Urbanismo - UNISUL, Palhoça, 2005. MINISTÉRIO DA SAÚDE, SECRETARIA EXECUTIVA. Legislação em saúde mental 1990-2002. 3. ed. revista e atualizada. Brasília: Ministério da Saúde, 2002.

RHEINGANTZ, P. A.; AZEVEDO, G. A.; BRASILEIRO, A.; ALCANTARA, D.; QUEIROZ, M. Observando a Qualidade do Lugar: Procedimentos para a Avaliação pósocupação. Ed. Proarq Programa de Pós-graduação em Arquitetura da Universidade Federal do Rio de Janeiro, 2008.

SECRETARIA MUNICIPAL DE SAÚDE E SANEAMENTO. Ofício $\mathbf{n}^{\circ} \mathbf{0 7 6}$, de $\mathbf{5}$ de março de 2018. Balneário Camboriú, p.1-5, 5 mar. 2018. 